\title{
Ultrasound in the Management of Pleural Disease
}

\section{ABSTRACT}

\section{1 - Introduction}

Pleural disease encompasses a large range of conditions, is a common presentation to the acute medical take and often requires comprehensive investigation and treatment. Ultrasound is well recognised as a useful investigative tool in pleural disease especially in the field of pleural effusion, pleural thickening and interventional procedures. Thoracic ultrasound (TUS) has gained widespread use by physicians as evidence has shown a reduced rate of complications when performing pleural procedures with ultrasound guidance.

\section{2 - Areas covered}

This article will review studies assessing the role of TUS in the management of pleural disease and examine ongoing research into how TUS could advance our knowledge and understanding over the next decade.

\section{3 - Expert commentary}

Physician lead thoracic ultrasound has become commonplace over the last decade, and now represents a minimum standard of safety in conducting the majority of "bedside" pleural procedures. The current evidence points to important diagnostic and procedural roles of the use of bedside thoracic ultrasound. In the future, research developments are likely to lead to the use of thoracic ultrasound in prognostication, targeted treatment and understanding pathogenesis in pleural disease.

\section{BACKGROUND}

Pleural disease affects over 3,000 people per million population every year(1) and includes conditions such as pleural infection, effusion, malignant pleural disease and pneumothoraces. The assessment of these conditions requires a combination of clinical and radiological expertise in which ultrasound now plays a key role.

Until recently, ultrasound was primarily performed by radiologists. In 2008, a National Patient Safety Agency (NPSA) report showed that there was an unacceptable level of harm during the "blind" (nonimage guided) insertion of intercostal drains(2) for pleural fluid conditions, and recommended using bedside ultrasound to identify a safe area for drain placement. This led to the widespread employment of thoracic ultrasound by respiratory physicians, and achieving Level One Royal College of Radiology (RCR) Ultrasound competence is now a training requirement in the curriculum for respiratory registrars in the U.K(3).

Over the last decade, the utilisation of TUS has expanded as physicians have recognised the advantage of its use in the diagnosis and management of numerous respiratory conditions. TUS can be used to examine superficial and deeper structures in the thorax, and has a greater sensitivity compared with chest radiograph in identifying the presence of pleural fluid (4). TUS is increasingly 
recognised as an imaging modality with high sensitivity for identification of malignant pleural involvement, pleural thickening and septations within pleural effusions. There is now a wider scope for respiratory physicians to perform procedures which were traditionally performed by radiologists, such as ultrasound-guided pleural biopsies.

The field of TUS continues to move forward in areas such as determining the need for intervention in pleural infection, identifying trapped lung and predicting pleurodesis success. As diagnostic algorithms are created and physicians' ultrasound training becomes more advanced, we may be able to determine the correct management for patients before the first procedure is performed. This article will review the past, present and future course of TUS and highlight areas of future research.

\subsection{Imaging modalities in pleural disease}

The three most common imaging modalities in pleural disease are chest radiograph, TUS and computed tomography (CT). Chest radiography is a useful tool in screening for pleural disease as it is widely available, but does not often provide adequate information on underlying aetiology and is relatively insensitive for a number of conditions. Chest radiographs remain a key investigation in the management of pneumothorax; the British Thoracic Society guidelines (5) are based on size of pneumothorax as measured at the hilum on chest radiograph. Although this is a useful distinction in clinical practice, it is recognised that using chest radiographs to predict the volume of pneumothorax has limitations(5).

Chest radiography has significant limitations when assessing pleural fluid. More than $200 \mathrm{mls}$ of pleural fluid must be present to be detected on an erect posteroanterior chest radiograph(6), and it is not possible to reliably distinguish between pleural fluid, dense consolidation and a raised hemidiaphragm(7). Chest radiographs are unable to differentiate free flowing from septated effusion, or pleural fluid from pleural thickening. Chest radiographs therefore clearly have their place in identifying pleural disease and are valuable in the management of pneumothoraces but are a relatively blunt tool in the diagnostic and therapeutic pathway as a whole.

Thoracic CT provides a very significant amount of anatomical and 3-dimensional information, but is associated with specific advantages and disadvantages. They are associated with significant radiation dose (although this is constantly reducing with higher speed scanners), require the patient to be transported and often require a number of breath holds to obtain accurate images. They have the advantages of providing a reproducible image of the entire thorax, precise 3-dimensional relationships of tissues within the thorax and accurately demonstrate the position and depth of the abnormality, even if there is a combination of air, fluid and tissue. CT is thus the best imaging modality for the lung parenchyma and are vital when information is needed from both the lung parenchyma and pleural surface.

Studies have demonstrated that enhancement of the parietal pleura on CT is sensitive and specific in diagnosing pleural inflammatory conditions, the most common cause of which is pleural infection(8). CT scans reliably differentiate empyema from lung abscesses, with empyema classically demonstrating the 'split pleura' sign after a period of inflammation. In addition, the collection's 
shape, effect on the lung and relationship to the chest wall are also useful in differentiating these conditions(9).

In the diagnosis of malignant effusions, CT features such as pleural nodularity, pleural thickening more than $1 \mathrm{~cm}$ and circumferential pleural disease are highly sensitive for the diagnosis. (10). A large retrospective review performed in patients who underwent a CT scan prior to thoracoscopy found that using the $\mathrm{CT}$ criteria of nodular pleural thickening, mediastinal pleural thickening, parietal pleural thickening greater than $1 \mathrm{~cm}$ and circumferential pleural thickening, there was a $68 \%$ sensitivity and $78 \%$ specificity in predicting malignancy (11). CT confidently diagnoses pleural lipomas, are the gold standard for diagnosing pleural plaques and show classical features in rounded atelectasis(12), especially as the majority of change in this condition is lung parenchymal.

Ultrasound scanning is both operator and patient dependent. It is only possible to obtain images of areas where the probe can be placed and the ultrasound waves can penetrate. If the patient is unable to move freely, it may not be possible to scan the entire thorax and areas can be obscured by bone, tissue oedema, surgical emphysema and body habitus. An inexperienced operator may not obtain or record adequate images and can be misled by commonly produced artefacts in the scan. Ultrasound does however have significant advantages; these include a reasonably low cost once the equipment has been purchased, the lack of any radiation, the ability to take equipment to the patient and the "realtime" nature of the scan (13). This ability to provide information in real time at the bedside allows clinicians to make prompt decisions about ongoing care. This is especially important in critically unwell or unstable patients such as in the intensive care setting (14).

Multiple protocols have been developed to rapidly assess the most common causes of ventilator failure in a critical care setting (15). The ability of TUS to monitor fluid balance in critically unwell patients by noting the development of interstitial oedema has been demonstrated (16) and there is evidence that TUS aids in making a diagnosis of decompensated heart failure more accurately (17). TUS is the modality of choice when looking for pleural septations (18) and pleural thickening (19) but is most commonly used as the gold standard method of identifying pleural fluid and obtaining an appropriate anatomical site to perform pleural intervention(20).

In cases where ultrasound is able to provide the same information as $\mathrm{CT}$, it is advisable to use ultrasound due to the low cost and lack of radiation exposure. One great advantage to ultrasound is that it is now often performed by the clinician responsible for the patient's care, which means the clinician has control over how quickly the test is performed, and has the clinical knowledge to make further management decisions immediately. Using physician-led ultrasound as the initial test can save time and expense for radiology departments and leads to faster and more accurate patient management. While there is a place for all imaging modalities, the advances in ultrasound are the most exciting area for respiratory physicians.

\section{$\underline{2.2 \text { - Thoracic Ultrasound }}$}

An ultrasound image is created by a transducer receiving reflected ultrasound waves, or echoes, which are then constructed into an image displayed on a screen. An echo is produced at the interface between two materials, where the ultrasound waves are reflected to different extents. 
When this occurs, the majority of the ultrasound waves are reflected and a bright image is produced at the interface between the materials. If there is little density difference between the structures, most of the ultrasound waves will pass through, and thus the sonographic interface between the two materials will be less distinct. The classic example of significant reflectivity is at the diaphragm where there may be an interface between abdominal organs and fluid where pleural fluid is present(slide 1). The presence of pleural effusion is sonographically helpful as it provides an excellent acoustic window to view deeper structures and is the reason that TUS can provides clinically useful information. Air is a poor conductor of ultrasound waves and thus it is not possible to visualise any deeper structures when normal, aerated lung is present (slide 2 ).

Confusion in image interpretation may occur when a "black" (poorly echogenic) area on the sonographic image is interpreted only as pleural effusion. It should be possible to visualise deeper structures distal to the pleural effusion, whereas no such structures will be visible when an artefact (such as a rib shadow) is present (slide 3 ). As bones are dense, they reflect the majority of the ultrasound waves - a rib shadow thus appears black in the majority of the ultrasound image, because sound waves are reflected superficially, showing a bright white area at the top of the screen where the rib begins and a black area below this, where no further ultrasound waves have penetrated. Misinterpreting a rib shadow as pleural fluid is a common pitfall amongst inexperienced operators.

Ultrasound examinations are performed with a number of different probes with different frequencies, depending on the focus of the examination. TUS is performed using a usually curved probe with a frequency of $2-6 \mathrm{MHz}$. This permits good tissue penetration, allowing identification of deeper structures such as pleural effusions, atelectatic lung, abdominal organs and diaphragmatic pleura, but will provide less detail on superficial structures. Linear probes, at a frequency of 7-12 $\mathrm{MHz}$, can be used to examine more superficial structures in fine detail, including the chest wall, ribs, lymph nodes and the pleural interface. Higher frequency probes are particularly useful in the identification of pneumothorax (21).

There are three modes commonly used in TUS which have distinct clinical utility. Classical 2dimensional ultrasound is used to identify thoracic organs and, importantly, pleural fluid to help safely perform pleural procedures, known as B-mode (brightness mode). In B-mode, different parts of the image are shown as different levels of brightness, allowing the identification of structures and abnormalities. M-mode (motion mode) highlights one area and monitors this against a time axis, showing whether there is movement, and is used as an adjunct to B-mode (slide 4), especially in the detection of pneumothorax. Colour Doppler mode is used to demonstrated pleural fluid movement, and to identify vascular structures with blood flow. Colour Doppler mode is used once a conventional image has been obtained, to show movement with the image demonstrating red and blue signals which correspond to movement either towards or away from the probe(22). Colour Doppler can be used to find intercostal vessels (slide 5) and track their course between the rib to prevent injuries during thoracentesis, and to diagnose intercostal bleed by demonstrating a jet of fluid moving from the area around the pleura into the pleural space(23). The 'colour fluid sign' is present, even in small effusions, when respiratory movement causes the fluid to move within the effusion, thus creating a colour signal that implies a free flowing effusion(slide 6)(24). This can help differentiate between fluid and pleural thickening, but false negatives can occur in loculated effusion which are isolated from respiratory movement. There is ongoing research into newer techniques 
that may allow for differentiation of benign and malignant tumours, or identification of vascular anomalies which may extend the remit of TUS even further(24).

\section{$\underline{2.3 \text { - Untangling terminology }}$}

A range of terminology is employed when recording ultrasound findings which, is used to gather information to inform diagnosis. When a pleural effusion is being assessed, it can be described as echogenic, anechoic or non-echogenic and simple or complex, with or without septations or loculations. These terms are often used interchangeably but there are subtle differences.

Anechoic (or non-echogenic) effusions are found when no ultrasound waves are reflected in the fluid and will look completely black on the screen, which means that there are no particulates in the effusion. These are also known as simple effusions. (Slide 7)

Echogenic effusions demonstrate swirling or floating echoes which reflect the ultrasound waves, showing grey/white areas in the fluid. (Slide 8) These echoes represent variable amounts of fibrin strands, protein, blood or pus which are moved by the cardiac pulsation (25). The terms "septated", "loculated" and "complex" are often employed interchangeably and used to guide management, although there are little data to support this. Septated effusions are characterised by the visualisation of strands within the effusion (Slide 9); these can be free floating and thin or more advanced and able to divide the effusion into discrete pockets. A loculated effusion is characterised by discrete pockets of fluid, often in different areas of the hemithorax, which are completely separate from each other (Slide 10). These are usually diagnosed by CT as a comprehensive 3D image is required to inspect all areas where loculations may be present(26).

One system of nomenclature divides effusions into anechoic, complex non-septated, complex septated and homogenously echogenic(27). Complex non-septated effusions and complex septated effusions are both effusions with particulates, either with or without septations. Septations are describes as fibrin strands that can either float inside an effusion or form branches or webs(28). Homogenously echogenic effusions are most commonly due to either empyema or a haemothorax(29) and often have a speckled appearance (30) which can be difficult to distinguish from consolidated lung tissue.

The term "complex" pleural effusion has both a sonographic meaning and a clinical meaning. A sonographically complex effusion is one with a high cellular content, echoes in the fluid or septations (i.e visually complex). A clinical "complicated" pleural effusion is used in the context of pleural infection, in which a parapneumonic effusion is identified as having specific biochemical characteristics (i.e. non-purulent but either $\mathrm{pH}<7.2$ or glucose $<60 \mathrm{mg} / \mathrm{dl}$ ) (31) which are predictive of a complex clinical course. This diagnosis requires the urgent insertion of a chest drain to decrease morbidity and mortality (32). It is the latter definition of a complex pleural effusion that has been incorporated in guidelines as a management strategy (33), rather than the radiological definition. Using these terms interchangeably is misleading as a sonographically simple effusion can have a pH $<7.2$ and need prompt drainage to improve the patient's outcome. Likewise, a septated effusion does not always require drainage. 
A range of other terminology is used in reference to the normal lung movement, including "lung sliding", "lung pulse" or "curtain". Lung sliding is seen when the visceral and parietal pleura glide over each other (34). This is usually present in when there is only a small amount of fluid allowing the pleura to slide smoothly. Although the visceral pleura can be identified it is not possible to ascertain detailed information about the lung parenchyma underneath. When there is an absence of lung sliding, it can be a sign of a pneumothorax, successful pleurodesis or malignant infiltration. The lung pulse is a rhythmic movement of the visceral pleural caused by transmission of the cardiac pulse (34) and can most clearly be seen in collapsed or atelectatic lung, where there is a continuous column of matter for the pulse to be transmitted through. It can be seen in pleurodesed lung or on breath hold, but effectively rules out pneumothorax. Lastly, the diaphragm is identified through the curtain effect in healthly lung. The interface between soft tissue and aerated lung is less marked than the interface between soft tissue and pleural fluid. This means that the diaphragm will not normally be visible throughout its length as a bright white line in a healthy individual. When the upper abdomen is visualised and the patient takes a breath in, the lung displaces the structures below as the diaphragm moves down, which looks like a curtain of black (Slide 11). This indicates that the lung base is moving freely and that there is no pleural fluid.

\section{$\underline{3 \text { - PLEURAL EFFUSIONS }}$}

There are many pieces of information that can be gleaned from TUS when assessing pleural effusion. Estimated volumes can be calculated, the complexity of an effusion can suggest an appropriate course of action in infection, and ultrasound can be used to identify safe sites for interventions.

\section{$\underline{3.1 \text { - Transudate vs exudate }}$}

A significant number of studies have considered whether ultrasound can determine whether a pleural effusion is a transudate or exudate. An analysis of 320 pleural effusions showed that all transudates were anechoic but exudates could be echoic or anechoic (25). It also found that complex septated and non-septated, and homogenously echogenic effusions were always exudates $(p<0.01)$.

\section{$\underline{3.2 \text { - Depth and Volume Calculations }}$}

There are numerous methods of calculating the volume of a pleural effusion but, as yet, none have been validated for use in routine clinical practice. A study estimating pleural fluid volume in ventilated patients demonstrated that the maximal distance between visceral and parietal pleura in millimetres multiplied by 20 predicts volume of effusion to within $160 \mathrm{mls}$ (35). Numerous other studies in acutely unwell or perioperative patients have produced similar calculations and reliable results $(36,37)$, but this has not been validated in routine respiratory care.

Decisions regarding pleural intervention are based on clinical presentation rather than volume of fluid in the pleural effusion. Estimations of small, moderate or large effusions are regularly performed by clinicians, but as decisions regarding management of effusion do not relate to estimate of volume, a simple estimate of size (small, moderate or large) is usually sufficient, with a single measurement of size for documentation purposes. The authors classify effusion size on the basis of vertical height with the patient sitting follows: small 1 or $<1$ rib space, moderate 2-3 rib spaces, large $>4$ rib spaces. 


\section{$\underline{3.3 \text { - Pleural infections }}$}

The rate of pleural infection is increasing $(38,39)$ and it is associated with significant morbidity and mortality despite advances in treatment $(40,41)$. The most important use of TUS in pleural infection is to deliver information so pleural interventions can be appropriately timed and completed. A study investigating whether TUS can predict the biochemical complexity of an effusion did not show any correlation between sonographic appearances and Light's stages of an exudative effusion (42). There are studies which show that TUS can still be helpful in the diagnosis and management of infected pleural space. A recent study showed that TUS had a sensitivity of $69 \%$ and a specificity of $90 \%$ in predicting a biochemically complex pleural effusion (43). The authors conclude that TUS is a good 'rule in' test to determine which patients will need a chest drain inserted but is not reliable enough to rule out a biochemically complex effusion from the ultrasound appearance alone.

A study of patients in a medical ITU with a persistent fever and pleural effusion showed that TUS can be used to identify which patients need prompt thoracentesis to rule out an empyema (44). 118 procedures were performed in 94 patients and 15 empyemas were identified. No patients with 'complex non-septated and relatively nonhyperechoic' or anechoic effusions had an empyema. Therefore, the authors argue that thoracentesis can be avoided in these patients. Conversely, those with 'complex septated and relatively hyperechoic patterns', 'complex septated' or 'homogeneously echogenic' patterns had a rate of empyema of $43 \%$ (44). This study used the definition of an empyema as either frank pus or microbiological isolation of an organism rather than pleural $\mathrm{pH}$, meaning that the study result may not translate directly into routine clinical practice, but does highlight the positive predictive value of TUS in diagnosis.

Studies have shown that a non-septated complex effusion has a higher likelihood of successful drainage with small bore catheters than a septated complex effusion, which has an overall poorer prognosis along with a higher mortality (45). Patients with septated effusions have been reported as having a higher likelihood of requiring intrapleural fibrinolytic therapy or surgical intervention than those with non-septated effusions (28). These findings need to be interpreted with caution as many of the trials were retrospective and unblinded. All such studies suggest that TUS may have a role in determining management in complex pleural effusions, but large scale prospective trials are needed to validate these results. Until such studies are conducted, diagnostic thoracentesis remains the first and necessary management step in suspected pleural infection.

\section{4 - Pleural malignancy}

There are a number of ultrasound features suggestive of a malignant aetiology in pleural effusion. A study using the validated CT criteria for malignant effusions was performed to review the predictive value of certain ultrasound criteria when identifying malignant effusions (46). Using the presence of one of pleural thickening greater than $1 \mathrm{~cm}$, diaphragmatic nodularity, parietal pleural nodules or hepatic metastases on TUS, there was a $100 \%$ specificity and a $73 \%$ sensitivity in predicting a malignant pleural effusion (MPE). A study in the United States produced similar results and cited that pleural nodularity was the most significant predictor of malignancy but also included some benign features such as the lung air bronchogram sign (47). In one study of patients with a previously diagnosed malignancy, $81.8 \%$ of those with an echogenic swirling pattern had an MPE confirmed but $48 \%$ of patients without the swirling pattern were confirmed to have an MPE (48), suggesting that the presence of swirling is suggestive, but not specific, to MPE. Pleural and 
diaphragmatic nodularity were consistently found to be a feature of a MPE (Slide 12) $(46,47)$, although no feature had a high enough sensitivity or specificity to replace cytological examination.

\section{$\underline{3.5 \text { - Pleural thickening and nodularity }}$}

Focussed ultrasound of the chest wall and pleura can be used to demonstrate pleural thickening, nodularity and malignant invasion. CT has been shown to have poor sensitivity in detecting malignant chest wall invasion and comparative studies have shown that TUS is superior $(49,50)$ and can predict malignant involvement of the pleura and chest wall $(51,52)$. US Colour Doppler can be used to differentiate between small pleural effusions and pleural thickening with $89 \%$ sensitivity and $100 \%$ specificity (53).

\section{$\underline{4-\text { Pneumothorax }}$}

A pneumothorax can be diagnosed by TUS by both B-mode and M-mode. In B-mode, there is an absence of lung sliding, demonstrated by the absence of movement at the level of the pleura during inspiration. M-mode demonstrates the classical "seashore sign" in normal lung (Slide 13). In the presence of lung sliding, the ultrasound image splits into two halves with the half closest to the probe representing structures up to and including the parietal pleura. This area does not move and therefore produces horizontal lines (the "sandy beach"). After the parietal pleura, when lung sliding is present, there is movement which produces a rougher US image. This clear interface demonstrates the difference in motion and therefore lung sliding. In patients with a pneumothorax, there is an absence of lung sliding and so the ultrasound field only produces horizontal lines(Slide 14) which is known as the barcode sign. It should be noted that in patients with severe emphysema/hyperinflation, lung sliding is often not identified even in the absence of pneumothorax, as there can be very little movement of the lung during respiration.

TUS is not routinely used to aid pleural interventions in patients with pneumothoraces. A recent study has investigated whether TUS can be used to identify a safe position for intervention in the case of loculated pneumothoraces (54), using ultrasound to identify areas of absence of lung sliding where an intercostal drain could be placed. This is not routine practice, although the authors routinely use thoracic ultrasound in pneumothorax intervention to identify the hemidiaphragm position and measure the thickness of the chest wall.

\section{$\underline{5 \text { - Ultrasound-guided procedures }}$}

In 2008, the National Patient Safety Agency reported on incidents between January 2005 and March 2008 involving harm caused by chest drain placement, demonstrating 12 episodes of death (2). A large body of evidence has now shown that complications can be reduced with the use of thoracic ultrasound for fluid drainage procedures (55-58), and this has led to a change in treatment guidelines (20).

Perhaps the most important study in this area asked a group of clinicians to identify a safe site for aspiration in patients with a pleural effusion using clinical examination. Clinicians were only able to identify a site in $67 \%$ of patients and in $15 \%$ of these, the site identified was inaccurate once TUS scanning had been performed(59). The use of TUS is associated with fewer complications such as 
pneumothoraces (57) and unsuccessful aspiration attempts (60), and TUS has been shown to be especially effective in obtaining samples from small pleural effusions (61). A large observational cohort study reviewed 61,261 thoracentesis records and showed that ultrasound guidance reduced the risk of pneumothoraces by $19 \%$ (55).

The evidence that supports the use of TUS is overwhelming in multiple scenarios, such as differentiating between consolidation and fluid, especially in those with a complete white out (62). Although current guidelines suggest inserting a chest drain into a large pleural effusion is acceptable without TUS guidance (the so called "white out"), this is not recommended and the authors advocate ultrasound for all fluid based procedures. This is due to the fact that a white out not caused by pleural effusion can be easily misdiagnosed on chest radiograph, or underlying abnormalities, such as an enlarged heart or raised hemidiaphragm, could be missed, both of which could lead to visceral injury if not identified prior to intervention(63).

Physician-led TUS has been introduced to mainstream clinical practice over the last decade. A study comparing the major complication rate of respiratory physician-led TUS for diagnosis and intervention in pleural disease and found that the rates were comparable to published literature (64) which previously focussed on TUS performed by radiologists. The overwhelming evidence of increased safety has led to a change in practice which was incorporated into the 2010 British Thoracic Society Pleural Procedures and Thoracic Ultrasound Guidelines (20).

Ultrasound has been utilised in finding an appropriate site for pleural biopsies, especially in the case of malignancy where the pleural changes are less uniform (65). Image-guided biopsies have a higher diagnostic yield than blind pleural biopsies (66) and ultrasound is safe, mobile and does not involve radiation exposure. A retrospective review of a physician-based ultrasound guided biopsy service demonstrated sufficient tissue for histological diagnosis in $94 \%$ of patients, with only one case of false negative biopsy (2\%) over a median follow-up of 16 months (67). A study assessing the feasibility and safety of chest physicians performing US-guided cutting needle biopsies demonstrated a high success rate and minimal complication in pleural lesions of $20 \mathrm{~mm}$ or above (68). This technique is especially useful in patients who are frail and those in whom is it not possible to perform thoracoscopy due to lung tethering or loculated effusion.

Where physicians are trained in US-guided pleural biopsies and thoracoscopy, it is possible to convert from thoracoscopy to ultrasound guided biopsy in the same sitting, if the former is unable to be performed. This prevents the patient having to attend for a further procedure (67). With regards to guided pleural and peripheral lung biopsies, TUS is comparable to CT and TUS may be superior to $\mathrm{CT}$ in complication rates such as post-procedure pneumothoraces (69). US-guided pleural biopsies are therefore an effective and safe way of obtaining a diagnosis, especially in patients who cannot have a thoracoscopy, in the hands of trained physicians.

\section{$\underline{6-\text { FUTURE DIRECTIONS }}$}

\section{$\underline{6.1 \text { - Identification of intercostal arteries }}$}

Trauma to the intercostal artery can be a serious complication of pleural procedures. Identification of the artery though ultrasound TUS is not currently routine in clinical practice. A study of 50 
patients comparing TUS to CT showed that the intercostal arteries that are unshielded by a rib can be accurately identified with a portable ultrasound machine (70).

Ultrasound may be used to identify intercostal bleeding after a pleural intervention. One case report described a "pulsatile plume of highly echogenic material" flowing into the pleural space(23) in such a situation, which may be useful in the early identification of complications, thus altering immediate management. Routine TUS post-procedure may also show a rapid increase in echogenic fluid, which increases the clinical suspicion of an intrapleural bleed.

\section{$\underline{6.2 \text { - Trapped lung and pleurodesis }}$}

TUS may provide information on whether a patient will have non-expandable lung. It is widely recognised that trapped or entrapped lung will not expand fully once pleural fluid has been drained (71) which means that subsequent pleurodesis is likely to fail. A study using M-mode and speckle tracking imaging (STI), which is a technique more commonly used in echocardiography, investigated whether these sonographic techniques could predict non-expandable lung (72). The hypothesis is that non-expandable, or trapped, lung has less movement as a result of the cardiac impulse than normal lung. The results showed that both M-mode and STI were $85 \%$ specific but only $50 \%$ and $71 \%$ sensitive respectively in detecting non expandable lung (72). There are ongoing studies to determine whether it is possible to determine which patients have achieved pleurodesis after the instillation of sterile talc to promote early removal of intercostal drains after pleurodesis and therefore shorten hospital stays (ISRCTN 16441661).

\section{$\underline{6.3 \text { - Contrast-enhanced US }}$}

Contrast-enhanced ultrasound (CEUS) has been effectively introduced in the field of liver disease for over a decade $(73,74)$. A key advantage is being able to view an area in multiple phases of contrast enhancement without subjecting the patient to large amounts of radiation. This has been especially useful in the liver using arterial phase, portal phase and late phase to determine whether liver lesions are malignant (74). Contrast agents have been found to be safe (75), are not nephrotoxic and no monitoring of renal function is needed (76), with a guideline on non-hepatic use of CEUS was published in 2011 (77). In the lung, CEUS can be used to identify abscesses or necrotic areas due to absence of contrast uptake and a tumour will normally have late enhancement as opposed to pneumonia which will have early enhancement $(78,79)$. There is a scope for using CEUS when performing pleural biopsies to avoid areas of necrosis, as shown by absence of contrast, to increase the likelihood of obtaining a viable specimen.

\section{$\underline{7-\text { CONCLUSIONS }}$}

Since the introduction of widespread physician-led TUS in the field of respiratory medicine, the remit of practice has expanded exponentially. TUS has been conclusively shown to increase diagnostic yield and safety when performing pleural procedures and national guidelines now advocate the use of TUS when performing any intervention with pleural fluid. Many studies have been published on the use of TUS in distinguishing MPEs from benign aetiologies and identifying whether an effusion is likely to require drainage in the context of pleural infection. Using TUS as part of clinical assessment is valuable as certain characteristics, such as septations in a parapneumonic effusion, can lead a clinician to suspect that drainage will be required. However, TUS has not yet been found to be 
reliable enough to make a diagnosis on radiology alone. Likewise, there are multiple ultrasonographic indictors to suggest that an effusion is likely to be malignant but these are not yet sensitive or specific enough to replace pathological analysis, but may help to direct the early investigation pathway.

The remit of TUS in the hands of physicians is increasing with regards to performing pleural biopsies and identifying post-procedural complications. There may be utility in using TUS to identify patients with trapped lung who are therefore unlikely to benefit from an attempt at pleurodesis, or to guide when a drain could be removed after pleurodesis. The value of TUS is being realised by other specialities, such as critical care, in the assessment of fluid status and acute lung pathologies. This is partly due to the ease of movement of the scanners, so investigations can be taken to the patient which is safer than transporting critically ill patients. Newer techniques are being introduced, such as contrast-enhanced ultrasound, which may expand the remit of TUS further. If the advances that have occurred over the last decade continue, in the future training in thoracic ultrasound may become as important as use of the stethoscope for respiratory and pleural practice.

\section{$\underline{8 \text { - EXPERT COMMENTARY }}$}

Physician led thoracic ultrasound has been introduced into routine clinical practice over the last decade, driven by an urgent need to improve basic safety of simple pleural procedures. The requirement for thoracic ultrasound for procedures has led to pleural procedures being performed increasingly by specialists. Delivering training in ultrasound to meet these standards has been challenging, but is now part of the respiratory training curriculum in the U.K. It is likely that a wider range of physicians will gain basic knowledge in thoracic ultrasound, but that pleural specialists will gain a deeper level of knowledge and will provide a referral service for more complex cases and procedures.

Thoracic ultrasound is an excellent point of care test, acting as an adjunct to clinical decision making, much like the physical examination. When considering which pleural interventions to perform, it is vitally important to understand the clinical context, and these factors, supported by ultrasound findings, can help decide whether to perform a diagnostic or therapeutic thoracentesis, chest drain insertion or allow earlier identification of patients with suspected malignancy so further imaging can be organised.

The key weaknesses in current clinical management are the requirement for aspiration to reliably identify those patients with established pleural infection, and the requirement for large volume aspiration to establish which patients have trapped lung, and which with malignant effusion will have a suitable symptomatic response. Identification of intercostal vessels with ultrasound is also within the scope of physicians but is often underused. Studies have shown that it is possible to reliably identify the vessels, which would provide further information about the risks and benefits of any given intervention. In the future, this should be incorporated into TUS training and may help earlier identification of possible complications.

An advanced ultrasound protocol is currently being studied in Oxford aiming to identify patients with trapped lung. This uses M-mode to assess transmission of the cardiac pulsation through atelectatic 
lung tissue as a surrogate marker for lung compliance. If this protocol accurately predicts trapped lung, patients could be directed towards appropriate therapies such as IPC insertion rather than attempting pleurodesis.

TUS is gaining increasing utility as a point of care test in other disciplines such as intensive care and renal medicine. The fluid status of a patient is vitally important in the management of these patients and there are on-going studies assessing the reliability of TUS. The ability to differentiate between acute pathologies with similar presentations is likely to lead to earlier effective interventions and therefore improved outcomes. This has potential clinical importance in specialities such as acute and emergency medicine. If on-going studies demonstrate patient outcomes can be improved by point of care ultrasound, there is the potential for training curriculums to include training in various aspects of thoracic ultrasound.

\section{9 - FIVE YEAR VIEW}

With on-going research studies, it is possible that sonographic parameters may allow us in the future to identify patients who require drainage or will have a trapped lung sonographically, shortening the treatment pathway and potentially preventing unnecessary procedures.

There is increasing evidence to show that ultrasound can predict trapped lung, identify septations which could predict a more complex clinical course and may be useful in differentiating between a malignant and non-malignant effusion. As these techniques become validated the diagnostic pathways for these patients may change.

\section{0 - KEY ISSUES}

1. Physician led thoracic ultrasound has become commonplace over the last decade, due to a National Patient Safety report highlighting an increased risk to patients without ultrasound guidance.

2. Ultrasound is superior to a chest radiograph in identifying pleural fluid and it can also differentiate between fluid, consolidation and underlying organs.

3. Physicians now use ultrasound to identify the "complexity" of effusions such as demonstrating echoes, swirling or septations.

4. Studies have shown that physicians can reliably identify intercostal vessels which will further improves the safety of pleural procedures.

5. Ongoing work includes using ultrasound to predict trapped lung prior, identifying which patients have achieved successful pleurodesis and investigating the role of contract enhanced ultrasound. These techniques will enhance the use of ultrasound in the diagnostic pathway in the future.

1. Light RW LY. Textbook of pleural diseases, 2nd ed. . London: Arnold Hodder; 2008.

2. Agency NPS. Rapid Response Report NPSA/2008/RRR003: Risks of Chest Drain Insertion. London, National Health Service; 2008.

3. Radiologists TRCo. Ultrasound training recommendations for medical and surgical specialties neLTRCoR, 2012. 
4. Lichtenstein D, Goldstein I, Mourgeon E, Cluzel P, Grenier P, Rouby JJ. Comparative diagnostic performances of auscultation, chest radiography, and lung ultrasonography in acute respiratory distress syndrome. Anesthesiology. 2004;100(1):9-15.

5. MacDuff A, Arnold A, Harvey J. Management of spontaneous pneumothorax: British Thoracic Society Pleural Disease Guideline 2010. Thorax. 2010;65 Suppl 2:ii18-31.

6. Blackmore CC, Black WC, Dallas RV, Crow HC. Pleural fluid volume estimation: a chest radiograph prediction rule. Academic radiology. 1996;3(2):103-9.

7. Sperandeo M, Filabozzi P, Varriale A, Carnevale V, Piattelli ML, Sperandeo G, et al. Role of thoracic ultrasound in the assessment of pleural and pulmonary diseases. Journal of ultrasound. 2008;11(2):39-46.

8. Waite RJ, Carbonneau RJ, Balikian JP, Umali CB, Pezzella AT, Nash G. Parietal pleural changes in empyema: appearances at CT. Radiology. 1990;175(1):145-50.

9. Stark DD, Federle MP, Goodman PC, Podrasky AE, Webb WR. Differentiating lung abscess and empyema: radiography and computed tomography. American Journal of Roentgenology. 1983;141(1):163-7.

10. Traill ZC, Davies RJ, Gleeson FV. Thoracic computed tomography in patients with suspected malignant pleural effusions. Clinical radiology. 2001;56(3):193-6.

11. Hallifax RJ, Haris M, Corcoran JP, Leyakathalikhan S, Brown E, Srikantharaja D, et al. Role of CT in assessing pleural malignancy prior to thoracoscopy. Thorax. 2015;70(2):192-3.

12. Helm EJ, Matin TN, Gleeson FV. Imaging of the pleura. Journal of magnetic resonance imaging : JMRI. 2010;32(6):1275-86.

13. Turner JP, Dankoff J. Thoracic ultrasound. Emergency medicine clinics of North America. 2012;30(2):451-73, ix.

14. Inglis AJ, Nalos M, Sue KH, Hruby J, Campbell DM, Braham RM, et al. Bedside lung ultrasound, mobile radiography and physical examination: a comparative analysis of diagnostic tools in the critically ill. Critical care and resuscitation : journal of the Australasian Academy of Critical Care Medicine. 2016;18(2):124.

15. Lichtenstein DA. BLUE-protocol and FALLS-protocol: two applications of lung ultrasound in the critically ill. Chest. 2015;147(6):1659-70.

16. Lichtenstein D. Fluid administration limited by lung sonography: the place of lung ultrasound in assessment of acute circulatory failure (the FALLS-protocol). Expert review of respiratory medicine. 2012;6(2):155-62.

17. Pivetta E, Goffi A, Lupia E, Tizzani M, Porrino G, Ferreri E, et al. Lung UltrasoundImplemented Diagnosis of Acute Decompensated Heart Failure in the ED: A SIMEU Multicenter Study. Chest. 2015;148(1):202-10.

18. Kearney SE, Davies CWH, Davies RJO, Gleeson FV. Computed Tomography and Ultrasound in Parapneumonic Effusions and Empyema. Clinical radiology. 2000;55(7):542-7.

19. McLoud TC, Flower CD. Imaging the pleura: sonography, CT, and MR imaging. AJR American journal of roentgenology. 1991;156(6):1145-53.

20. Havelock T, Teoh R, Laws D, Gleeson F. Pleural procedures and thoracic ultrasound: British Thoracic Society Pleural Disease Guideline 2010. Thorax. 2010;65 Suppl 2:ii61-76.

21. Tasci O, Hatipoglu ON, Cagli B, Ermis V. Sonography of the chest using linear-array versus sector transducers: Correlation with auscultation, chest radiography, and computed tomography. Journal of clinical ultrasound : JCU. 2016;44(6):383-9.

22. Evans DH, Jensen JA, Nielsen MB. Ultrasonic colour Doppler imaging. Interface focus. 2011;1(4):490-502.

23. Corcoran JP, Psallidas I, Ross CL, Hallifax RJ, Rahman NM. Always Worth Another Look? Thoracic Ultrasonography before, during, and after Pleural Intervention. Annals of the American Thoracic Society. 2016;13(1):118-21.

24. Hsu W-H. Clinical Applications of Color Doppler Ultrasound in Chest Medicine. Journal of Medical Ultrasound. 2007;15(3):141-51. 
25. Yang PC, Luh KT, Chang DB, Wu HD, Yu CJ, Kuo SH. Value of sonography in determining the nature of pleural effusion: analysis of 320 cases. AJR American journal of roentgenology.

1992;159(1):29-33.

26. McLoud TC. CT and MR in pleural disease. Clinics in chest medicine. 1998;19(2):261-76.

27. Hirsch JH, Rogers JV, Mack LA. Real-time sonography of pleural opacities. American Journal of Roentgenology. 1981;136(2):297-301.

28. Chen KY, Liaw YS, Wang HC, Luh KT, Yang PC. Sonographic septation: a useful prognostic indicator of acute thoracic empyema. Journal of ultrasound in medicine : official journal of the American Institute of Ultrasound in Medicine. 2000;19(12):837-43.

29. Prina E, Torres A, Carvalho CRR. Lung ultrasound in the evaluation of pleural effusion. Jornal Brasileiro de Pneumologia. 2014;40(1):1-5.

30. Soni NJ, Franco R, Velez MI, Schnobrich D, Dancel R, Restrepo MI, et al. Ultrasound in the diagnosis and management of pleural effusions. Journal of hospital medicine. 2015;10(12):811-6.

31. Porcel JM. Pleural fluid tests to identify complicated parapneumonic effusions. Current opinion in pulmonary medicine. 2010;16(4):357-61.

32. Heffner JE, Brown LK, Barbieri C, DeLeo JM. Pleural fluid chemical analysis in parapneumonic effusions. A meta-analysis. American journal of respiratory and critical care medicine.

1995;151(6):1700-8.

33. Davies HE, Davies RJ, Davies CW. Management of pleural infection in adults: British Thoracic Society Pleural Disease Guideline 2010. Thorax. 2010;65 Suppl 2:ii41-53.

34. Volpicelli G, Elbarbary M, Blaivas M, Lichtenstein DA, Mathis G, Kirkpatrick AW, et al. International evidence-based recommendations for point-of-care lung ultrasound. Intensive care medicine. 2012;38(4):577-91.

35. Balik M, Plasil P, Waldauf P, Pazout J, Fric M, Otahal M, et al. Ultrasound estimation of volume of pleural fluid in mechanically ventilated patients. Intensive care medicine. 2006;32(2):31821.

36. Usta $\mathrm{E}$, Mustafi M, Ziemer G. Ultrasound estimation of volume of postoperative pleural effusion in cardiac surgery patients. Interactive cardiovascular and thoracic surgery. 2010;10(2):2047.

37. Remerand F, Dellamonica J, Mao Z, Ferrari F, Bouhemad B, Jianxin Y, et al. Multiplane ultrasound approach to quantify pleural effusion at the bedside. Intensive care medicine. 2010;36(4):656-64.

38. Grijalva CG, Zhu Y, Nuorti JP, Griffin MR. Emergence of parapneumonic empyema in the USA. Thorax. 2011;66(8):663-8.

39. Farjah F, Symons RG, Krishnadasan B, Wood DE, Flum DR. Management of pleural space infections: a population-based analysis. The Journal of thoracic and cardiovascular surgery. 2007;133(2):346-51.

40. Maskell NA, Davies CW, Nunn AJ, Hedley EL, Gleeson FV, Miller R, et al. U.K. Controlled trial of intrapleural streptokinase for pleural infection. The New England journal of medicine. 2005;352(9):865-74.

41. Davies CWH, Kearney SE, Gleeson FV, Davies RJO. Predictors of Outcome and Long-term Survival in Patients with Pleural Infection. American journal of respiratory and critical care medicine. 1999;160(5):1682-7.

42. Kearney SE, Davies CW, Davies RJ, Gleeson FV. Computed tomography and ultrasound in parapneumonic effusions and empyema. Clinical radiology. 2000;55(7):542-7.

43. Svigals PZ, Chopra A, Ravenel JG, Nietert PJ, Huggins JT. The accuracy of pleural ultrasonography in diagnosing complicated parapneumonic pleural effusions. Thorax. 2016.

44. Tu CY, Hsu WH, Hsia TC, Chen HJ, Tsai KD, Hung CW, et al. Pleural effusions in febrile medical ICU patients: chest ultrasound study. Chest. 2004;126(4):1274-80. 
45. Chen $\mathrm{CH}$, Chen W, Chen HJ, Yu YH, Lin YC, Tu CY, et al. Transthoracic ultrasonography in predicting the outcome of small-bore catheter drainage in empyemas or complicated parapneumonic effusions. Ultrasound in medicine \& biology. 2009;35(9):1468-74.

46. Qureshi NR, Rahman NM, Gleeson FV. Thoracic ultrasound in the diagnosis of malignant pleural effusion. Thorax. 2009;64(2):139-43.

47. Bugalho A, Ferreira D, Dias SS, Schuhmann M, Branco JC, Marques Gomes MJ, et al. The diagnostic value of transthoracic ultrasonographic features in predicting malignancy in undiagnosed pleural effusions: a prospective observational study. Respiration; international review of thoracic diseases. 2014;87(4):270-8.

48. Chian CF, Su WL, Soh LH, Yan HC, Perng WC, Wu CP. Echogenic swirling pattern as a predictor of malignant pleural effusions in patients with malignancies. Chest. 2004;126(1):129-34. 49. Sperandeo M, Filabozzi P, Varriale A, Carnevale V, Piattelli ML, Sperandeo G, et al. Role of thoracic ultrasound in the assessment of pleural and pulmonary diseases. Journal of ultrasound. 2008;11(2):39-46.

50. Sugama Y, Tamaki S, Kitamura S, Kira S. Ultrasonographic evaluation of pleural and chest wall invasion of lung cancer. Chest. 1988;93(2):275-9.

51. Tahiri M, Khereba M, Thiffault V, Ferraro P, Duranceau A, Martin J, et al. Preoperative assessment of chest wall invasion in non-small cell lung cancer using surgeon-performed ultrasound. The Annals of thoracic surgery. 2014;98(3):984-9.

52. Caroli G, Dell'Amore A, Cassanelli N, Dolci G, Pipitone E, Asadi N, et al. Accuracy of transthoracic ultrasound for the prediction of chest wall infiltration by lung cancer and of lung infiltration by chest wall tumours. Heart, lung \& circulation. 2015;24(10):1020-6.

53. Wu RG, Yang PC, Kuo SH, Luh KT. "Fluid color" sign: a useful indicator for discrimination between pleural thickening and pleural effusion. Journal of ultrasound in medicine : official journal of the American Institute of Ultrasound in Medicine. 1995;14(10):767-9.

54. Skaarup SH, Folkersen BH. Ultrasound-assisted aspiration of loculated pneumothorax: A new technique. Journal of clinical ultrasound : JCU. 2016;44(5):326-30.

55. Mercaldi CJ, Lanes SF. Ultrasound guidance decreases complications and improves the cost of care among patients undergoing thoracentesis and paracentesis. Chest. 2013;143(2):532-8.

56. Patel PA, Ernst FR, Gunnarsson CL. Ultrasonography guidance reduces complications and costs associated with thoracentesis procedures. Journal of clinical ultrasound : JCU. 2012;40(3):13541.

57. Cavanna L, Mordenti P, Berte R, Palladino MA, Biasini C, Anselmi E, et al. Ultrasound guidance reduces pneumothorax rate and improves safety of thoracentesis in malignant pleural effusion: report on 445 consecutive patients with advanced cancer. World journal of surgical oncology. 2014;12:139.

58. Jones PW, Moyers JP, Rogers JT, Rodriguez RM, Lee YC, Light RW. Ultrasound-guided thoracentesis: is it a safer method? Chest. 2003;123(2):418-23.

59. Diacon $\mathrm{AH}$, Brutsche $\mathrm{MH}$, Soler M. Accuracy of pleural puncture sites: a prospective comparison of clinical examination with ultrasound. Chest. 2003;123(2):436-41.

60. Perazzo A, Gatto P, Barlascini C, Ferrari-Bravo M, Nicolini A. Can ultrasound guidance reduce the risk of pneumothorax following thoracentesis? Jornal brasileiro de pneumologia : publicacao oficial da Sociedade Brasileira de Pneumologia e Tisilogia. 2014;40(1):6-12.

61. Kohan JM, Poe RH, Israel RH, Kennedy JD, Benazzi RB, Kallay MC, et al. Value of chest ultrasonography versus decubitus roentgenography for thoracentesis. The American review of respiratory disease. 1986;133(6):1124-6.

62. Wu HD, Yang PC, Kuo SH, Luh KT. Ultrasonography in complete chest X-ray opacification of hemithorax. Taiwan yi xue hui za zhi Journal of the Formosan Medical Association. 1989;88(7):694-9.

63. Havelock T, Teoh R, Laws D, Gleeson F. Pleural procedures and thoracic ultrasound: British Thoracic Society pleural disease guideline 2010. Thorax. 2010;65(Suppl 2):i61-i76. 
64. Rahman NM, Singanayagam A, Davies HE, Wrightson JM, Mishra EK, Lee YCG, et al. Diagnostic accuracy, safety and utilisation of respiratory physician-delivered thoracic ultrasound. Thorax. 2010;65(5):449-53.

65. Koegelenberg CF, Diacon AH. Image-guided pleural biopsy. Current opinion in pulmonary medicine. 2013;19(4):368-73.

66. Rahman NM, Gleeson FV. Image-guided pleural biopsy. Current opinion in pulmonary medicine. 2008;14(4):331-6.

67. Hallifax RJ, Corcoran JP, Ahmed A, Nagendran M, Rostom H, Hassan N, et al. Physician-based ultrasound-guided biopsy for diagnosing pleural disease. Chest. 2014;146(4):1001-6.

68. Diacon AH, Schuurmans MM, Theron J, Schubert PT, Wright CA, Bolliger CT. Safety and yield of ultrasound-assisted transthoracic biopsy performed by pulmonologists. Respiration; international review of thoracic diseases. 2004;71(5):519-22.

69. Sconfienza LM, Mauri G, Grossi F, Truini M, Serafini G, Sardanelli F, et al. Pleural and peripheral lung lesions: comparison of US- and CT-guided biopsy. Radiology. 2013;266(3):930-5.

70. Salamonsen M, Dobeli K, McGrath D, Readdy C, Ware R, Steinke K, et al. Physicianperformed ultrasound can accurately screen for a vulnerable intercostal artery prior to chest drainage procedures. Respirology (Carlton, Vic). 2013;18(6):942-7.

71. Huggins JT, Doelken P, Sahn SA. Intrapleural therapy. Respirology (Carlton, Vic). 2011;16(6):891-9.

72. Salamonsen MR, Lo AK, Ng AC, Bashirzadeh F, Wang WY, Fielding DI. Novel use of pleural ultrasound can identify malignant entrapped lung prior to effusion drainage. Chest. 2014;146(5):1286-93.

73. Rettenbacher T. Focal liver lesions: role of contrast-enhanced ultrasound. European journal of radiology. 2007;64(2):173-82.

74. Nolsoe CP, Lorentzen T. International guidelines for contrast-enhanced ultrasonography: ultrasound imaging in the new millennium. Ultrasonography (Seoul, Korea). 2016;35(2):89-103.

75. Piscaglia F, Bolondi L. The safety of Sonovue in abdominal applications: retrospective analysis of 23188 investigations. Ultrasound in medicine \& biology. 2006;32(9):1369-75.

76. Jakobsen JA, Oyen R, Thomsen HS, Morcos SK. Safety of ultrasound contrast agents. European radiology. 2005;15(5):941-5.

77. Piscaglia F, Nolsoe C, Dietrich CF, Cosgrove DO, Gilja OH, Bachmann Nielsen M, et al. The EFSUMB Guidelines and Recommendations on the Clinical Practice of Contrast Enhanced Ultrasound (CEUS): update 2011 on non-hepatic applications. Ultraschall in der Medizin (Stuttgart, Germany : 1980). 2012;33(1):33-59.

78. Gorg C. Transcutaneous contrast-enhanced sonography of pleural-based pulmonary lesions. European journal of radiology. 2007;64(2):213-21.

79. Gorg C, Bert T, Kring R, Dempfle A. Transcutaneous contrast enhanced sonography of the chest for evaluation of pleural based pulmonary lesions: experience in 137 patients. Ultraschall in der Medizin (Stuttgart, Germany : 1980). 2006;27(5):437-44. 